\title{
Experimental study of high-performance cooling system pipeline diameter and working fluid amount
}

\author{
Patrik Nemec ${ }^{1, a}$, Milan Malcho $^{1}$, Peter Hrabovsky $^{1}$ and Štefan Papučík ${ }^{1}$ \\ ${ }^{1}$ University of Zilina, Faculty of Mechanical Engineering, Department of Power Engineering, Univerzitná 8215/1, 01026 Žilina, Slovakia
}

\begin{abstract}
This work deals with heat transfer resulting from the operation of power electronic components. Heat is removed from the mounting plate, which is the evaporator of the loop thermosyphon to the condenser and by natural convection is transferred to ambient. This work includes proposal of cooling device - loop thermosyphon, with its construct and follow optimization of cooling effect. Optimization proceeds by selecting the quantity of working fluid and selection of diameters vapour line and liquid line of loop thermosyphon.
\end{abstract}

\section{Introduction}

Demands on the dimensions of electronic components, more precisely their reduction, are an important factor for the proper function of these components. There are many requirements on increasing electronic components performance and currently unchanged its dimension or reduce them. In most cases this result in produce of greater heat amount and thus temperature too, what may endanger the reliability operation of electronic components or even the whole system. Therefore, it is necessary the generated heat remove to other places where it does not affect the electronic system. One from the many option of the electronic components cooling may be a cooling system based on evaporation and condensation of the working medium circumfluent in a closed loop. In this work is just such a cooling system designed to remove heat from the electronic components placed on the mounting plate. The heat removal to the ambient by this cooling system takes place without additional electrical energy, which means that it is going on a heat transfer by free convection $[1,2,3]$.

The closed loop thermosyphon may be visualised as a long hollow pipe, bent and the ends joined to form a continuous loop, filled with working fluid and orientated in a vertical plane. If the one side of the loop is heated and the other side cooled, the average density of the fluid in the heated side is less than in the cooled side. An essentially hydrostatic pressure difference, as a result of the thermally induced temperature gradient between the hot and the cold sides, rives the fluid flows around the loop. The 'buoyancy' force, as it is often termed, driving the fluid is in turn counteracted by an opposing frictional

\footnotetext{
a Corresponding author: patrik.nemec@fstroj.uniza.sk
}

force that tends to retard the flow [4]. A thermosyphon loop can transfer heat from the interior of a microelectronic system to a central location where space limitations are less stringent. The advantages that a thermosyphon system enjoys over a conventional refrigeration system include: (1) absence of moving parts leading to a more reliable system operation, (2) increased choices for selecting a working fluid compatible with microelectronics chips since it does not have to go through a refrigeration cycle, (3) reducing the decomposition rate of the working fluid as the higher temperatures at the compressor discharge in a vapour compression refrigeration system are not encountered, (4) clean operation as no oil is circulated through the system. In comparison to pool boiling systems employing vapour space condensation, a thermosyphon loop offers more flexibility in terms of providing a centralized condenser with different feed lines to individual evaporator stations. Further, with the addition of a liquid circulating pump in a thermosyphon loop, higher heat transfer coefficients associated with flow boiling systems could be realized [5].

\section{Cooling device construction}

The cooling device has been designed as a specific type of thermosyphon - loop thermosyphon whose operation depends on the position of the evaporation and condensation section. The condensation section has to be placed above the evaporation section. This means that the return of the condensate is affected by the gravity. The proposed model of the loop thermosyphon is heat removed into the surrounding by natural convection. 
Moreover, this type of cooling devices work on the evaporation and condensation principle of the working fluid in a closed loop, and therefore it do not need any mechanical elements or additional electric energy to drive the heat transfer medium. In the figure 1 is shown proposed model of the cooling device.

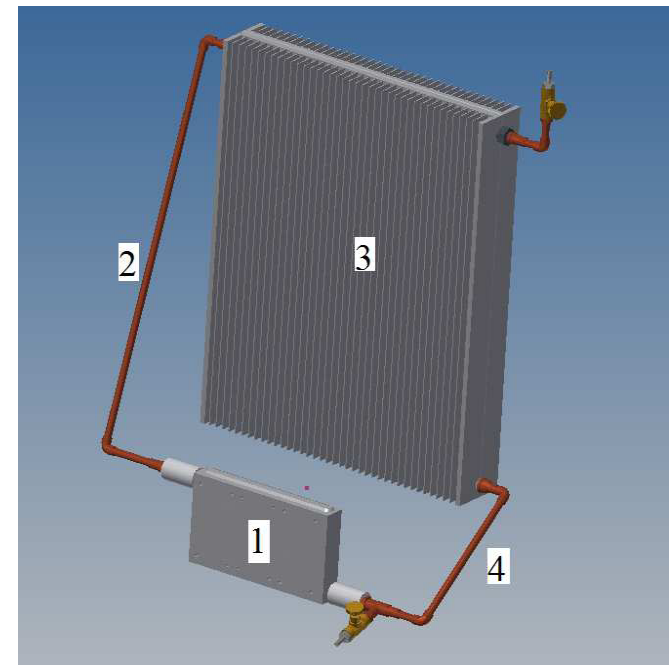

Figure 1. Model of cooling system, 1 - evaporator, 2 - vapour pipeline, 3 - condenser, 4 - liquid pipeline.

Main parts of the cooling device are:

- evaporator,

- condenser,

- pipeline,

- fill and vent valve,

- drain valve.

The evaporator enables on the base of a phase change (boiling) of the working fluid an intensive heat removal from its surface. It has to be constructed so that it will prevent the leakage of the working fluid, maintain pressure differences in all the walls and enable heat transfer from the electronic component into the working fluid as well as a suitable distribution of liquid and vapor phases of the working fluid. When choosing the material suitable for the construction of evaporator, it is necessary to pay attention to its thermokinetic characteristics. To provide a minimal temperature drop between a heat source and evaporator, the evaporator material must feature high thermal conductivity. To prevent escape of vapor, it should not be porous. The material should have high strength but, at the same time, it should be easily machineable and compatible with the working fluid [6]. The evaporator body shown in the figure 2 is a plate with dimensions $116 \times 206 \times 30 \mathrm{~mm}$. To provide the working fluid circulation there are two $12 \mathrm{~mm}$ openings drilled horizontally on the plate and connected with twenty one 6 $\mathrm{mm}$ vertical connecting channels. They provide the transport of heated fluid vapor from the bottom to the top section of the evaporator. On the outer contact surface of the evaporator and electronic component there is a groove with a mounted temperature sensor.

The condenser shown in the figure 3 is proposed so that it was possible removed heat in to the surrounding by natural convection. It is created from two alumina fin coolers with dimensions $400 \times 480 \mathrm{~mm}$ and fin height 45 $\mathrm{mm}$. The each cooler has on the back side along the length ninety $7.5 \mathrm{~mm}$ deep and wide vertical grooves, and on the top and bottom two $10 \mathrm{~mm}$ deep and wide horizontal grooves. The cooler are soldered together on the back side and through the grooves can the working fluid flow down to the evaporator. This robust condenser construction provides to the cooling device remove high heat fluxes loaded to the evaporator.

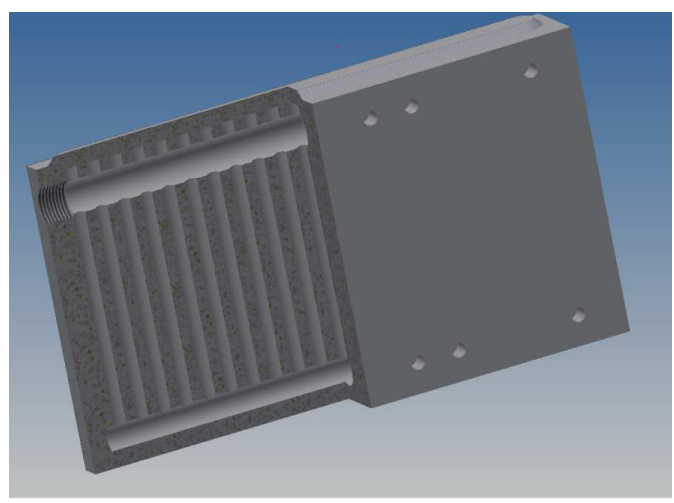

Figure 2. Evaporator of cooling system.

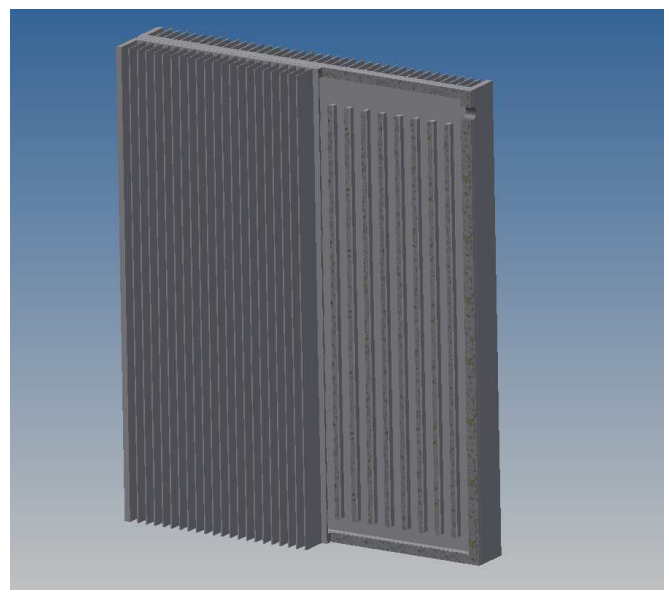

Figure 3. Condenser of cooling system.

The pipelines connected evaporator with condenser was designed as simple as possible, so that when the working fluid circulating in the loop has minimum pressure losses. The pipelines are made from copper material, due to its good installation properties and compatibility with almost all mediums used in heat pipe $[7,8]$.

One of the construction conditions of the cooling device is that the cooling system has to be electrically isolated from the electrical components. Therefore are on the input and output pipelines to the mounting plate inserted Teflon insulators that prevent a cooling system against possible electrical voltage breakdown. The other parts on the cooling device are needle valves. One is located at the top of the condenser, which is used as a fill and vent valve. At the bottom part of the evaporator at input is the second needle valve, which is used to discharge the working fluid [9].

The main objective in the cooling device design has been focusing on the maximal the heat flux transfer at high heat load of the mounting plate with electronic components. The cooling systems proposal is based on a 
comparison of current work dealing with electronic components cooling device investigation. In the figure 4 is a graphical comparison of cooling effect results different types of cooling devices with heat pipe technology. After results comparison has been chosen the cooling system was able remove the highest heat flux (LT with aluminium condenser).

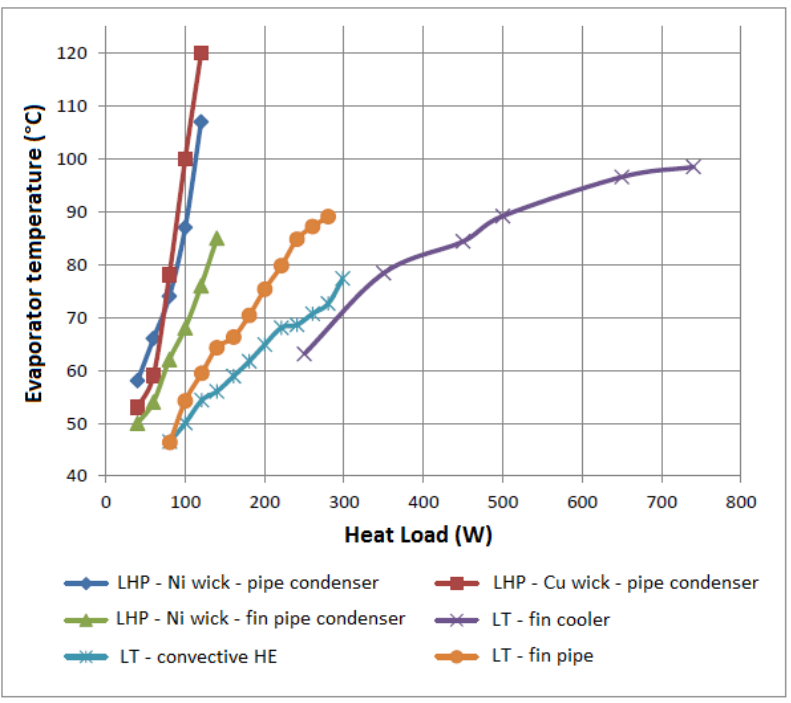

Figure 4. Comparison of cooling effect of different cooling devices with heat pipe technology.

\section{Working fluid amount option}

The scientific works declare that the proper percentage amount of the working fluid in loop thermosyphon is in the range of $40-60 \%$ of the total loop thermosyphon volume $[10,11,12]$. In this work was performed experiment to finding proper amount of the working fluid for the LT with working fluid percentage amount in the range of $20-70 \%$. As the working fluid was used ethanol. The pipeline diameter was $15 \mathrm{~mm}$. In the figure 5 are shown measurement values. In the graph is seen that the evaporator temperature curve of all percentage amounts, in addition to $70 \%$ are not very different. The differences are $\pm 1^{\circ} \mathrm{C}$, sometimes even less.

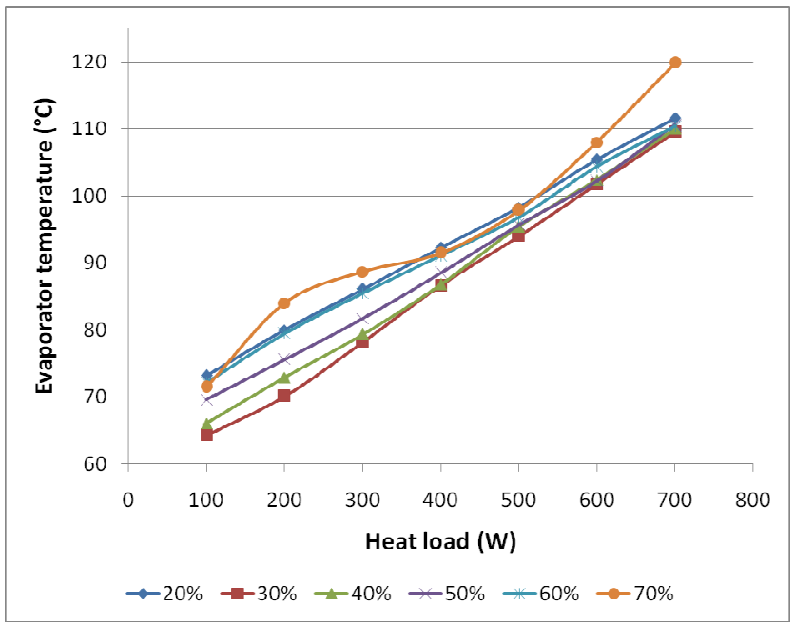

Figure 5. Influence of working fluid amount on cooling effect of cooling system.
In the figure 6 are shown results of evaporator temperature achieved at heat load $700 \mathrm{~W}$. Following the results in both graphs was optimal quantity of the working fluid in the cooling device 40 and $30 \%$ established. The choice of working fluid optimal quantity was realized on the basis of achieving the lowest evaporator temperature at the highest thermal load.

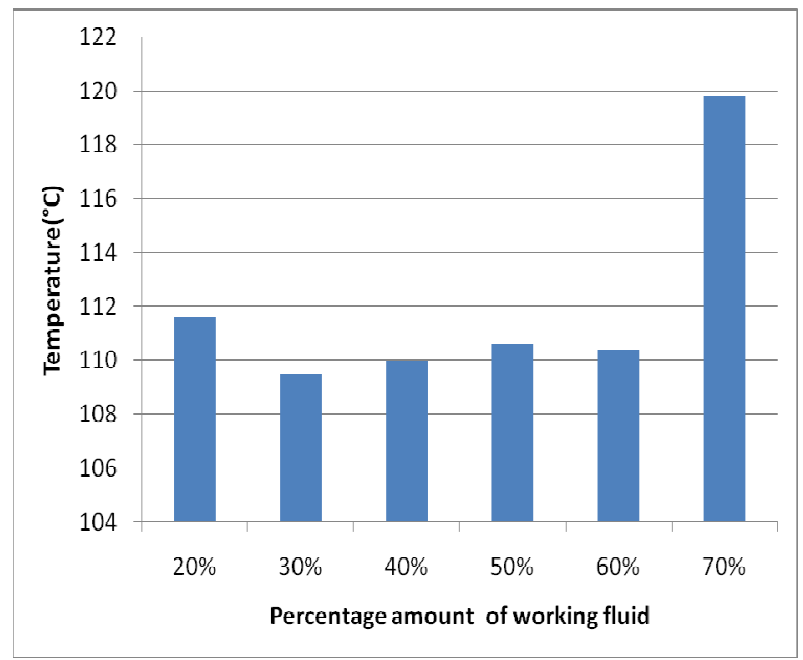

Figure 6. Evaporator temperature at heat load $700 \mathrm{~W}$

\section{Pipeline diameter option}

One of the possibilities affected on the amount of heat transferred through the LT is the option of the pipelines diameter. In the figure 7 are compared individual tested pipelines diameters of LT filled with the working medium ethanol. From the results is seen that the ethanol is compatible mainly with the large-diameter pipes. The size pipeline diameter $15 \mathrm{~mm}$ appears as a best choice for the LT with working fluid ethanol, when at the maximal heat load of the LT, the evaporator temperature did not rise above $110^{\circ} \mathrm{C}$.

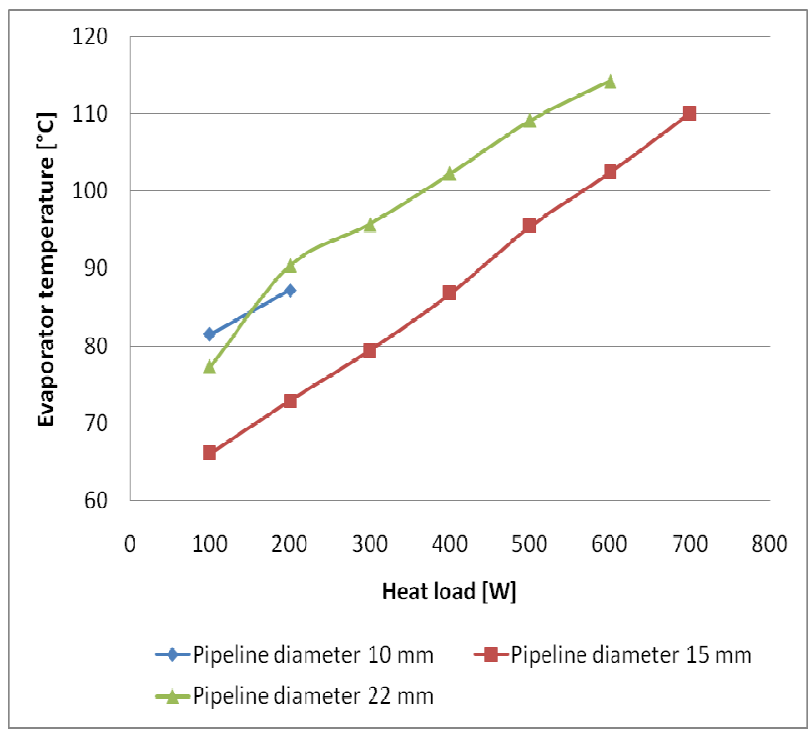

Figure 7. Influence pipeline diameter of cooling system on cooling effect. 


\section{Conclusion}

The correct function of the most electronic components depends on operating temperature range. In practice this mean, that the components and the equipments too has to be cooled, therefore the heat that is produced by using them has to be removed.

Aim of the work was to construct a cooling device and optimize the size of the pipeline diameter and quantity of working fluid, due to remove as much of the heat from the mounting plate of the electronic components. It has been found that a cooling device with a pipeline diameter of at least $15 \mathrm{~mm}$ working correctly, and that the size of pipeline diameter $10 \mathrm{~mm}$ and less is not suitable for this type of device, because with such a small pipeline diameter, this type of device cannot operate. From the measurements, it was found that the quantity of working fluid ideal for this type of cooling device is $40 \%$ or even $30 \%$ of its total volume, also. Performed measuring the quantity of heat removed at the maximum heat load found that the device made by us can remove sufficient heat so that the temperature of the electronic components fixed to the mounting plate does not exceed the limit of $120^{\circ} \mathrm{C}$ at the heat load $700 \mathrm{~W}$.

Other optimization of the designed cooling devices e.g. working fluid type option or condenser modification can overcome us detected limits.

\section{Acknowledgment}

This work is supported by the projects:

KEGA 029ŽU-4/2015 „Recovering heat from technological processes“" (33\%)

APVV 0577-10 "Cooling of power electronic systems by cooling cycles without mechanical drive" (34\%)

European Regional Development Fund and the Slovak state budget for the project "Research Centre of University of Zilina”, ITMS 26220220183 (33\%).

\section{References}

1. P. G. Peterson, An introduction to heat pipes modelling, testing and applications, (2010)

2. D. Reay, P. Kew and R. McGlen, Heat pipes Theory, design and applications, (2014)

3. C. Silverstein, Design and technology of heat pipes for cooling and heat exchangers, (2011)

4. C. Ruppersberg, R. T. Dobson, JESA, 18 (3), pp. 3240, (2007)

5. J. Hartenstine, R. Boner III, J. Montgomery, T. Semenic, ASME InterPAC, pp. 1-8 , (2007)

6. M. Holubčik, P. Durčanský, J. Jandačka and R. Nosek, The Sci. World J., 2014 (2014)

7. M. Vantúch, J. Hužvár and A. Kapjor, EPJ Web of Conf. 67, 02122, (2014)

8. R. Lenhard, J. Jandačka, AIP Conf. Proc., 1558, pp. 2138-2141, (2013)

9. M. Patsch, A. Čaja, EPJ Web of Conf., 95, 02061, (2015)

10. M. Hamdan, E. Elnajjare, World Acad. of Sci., Engineer. And Tech., 3, pp. 161-167, (2009)
11. D. Wolf, W. Bienert, Int. Conf. on Envir. Syst., 941576, (1994)

12. S. W. Kang, M. Ch. Tsai, Ch. S. Hsieh and J. Y. Chen, Tamkang J. of Sci. and Engineer., 13 (3), pp. 281-288, (2010) 